\title{
Existence results for impulsive differential equations with nonlocal conditions via measures of noncompactness
}

M. Mallika Arjunan ${ }^{a, *}$, V. Kavitha ${ }^{b}$, S. Selvi ${ }^{c}$

${ }^{a}$ Department of Mathematics, Karunya University, Karunya Nagar, Coimbatore- 641 114, Tamil Nadu, India.

${ }^{b}$ Department of Mathematics, Karunya University, Karunya Nagar, Coimbatore-641 114, Tamil Nadu, India.

${ }^{c}$ Department of Mathematics, Muthayammal College of Arts \& Science, Rasipuram- 637408, Tamil Nadu, India.

This paper is dedicated to Professor Ljubomir Ćirić

Communicated by Professor V. Berinde

\begin{abstract}
In this paper, we study the existence of integral solutions for impulsive evolution equations with nonlocal conditions where the linear part is nondensely defined. Some existence results of integral solutions to such problems are obtained under the conditions in respect of the Hausdorff's measure of noncompactness. Example is provided to illustrate the main result.(C)2012 NGA. All rights reserved.
\end{abstract}

Keywords: Impulsive differential equations, nondensely defined, noncompact measures, nonlocal conditions, integral solutions, semigroup theory.

2010 MSC: Primary 34A37, 34G10; Secondary 47D06.

\section{Introduction}

The purpose of this paper is to study the existence results for impulsive partial differential equations with nonlocal conditions in a real Banach space $X$ of the form:

$$
\begin{aligned}
u^{\prime}(t) & =A u(t)+f(t, u(t)), \quad 0 \leq t \leq b, \quad t \neq t_{i}, \\
u(0) & =g(u), \\
\Delta u\left(t_{i}\right) & =I_{i}\left(u\left(t_{i}\right)\right), \quad i=1,2, \ldots, p, \quad 0<t_{1}<t_{2}<\cdots<t_{p}<b,
\end{aligned}
$$

\footnotetext{
*Corresponding author

Email addresses: arjunphd07@yahoo.co.in (M. Mallika Arjunan), kavi_velubagyam@yahoo.co.in (V. Kavitha), sselvimaths@yahoo.com (S. Selvi)
} 
where $A: D(A) \subset X$ is a nondensely defined operator, $\Delta u\left(t_{i}\right)=u\left(t_{i}^{+}\right)-u\left(t_{i}^{-}\right), u\left(t_{i}^{+}\right), u\left(t_{i}^{-}\right)$denote the right and left limit of $u$ at $t_{i}$, respectively. $f, g$ and $I_{i}$ are appropriate functions to be specified later.

The theory of impulsive differential equations appears as a natural description of several real processes subject to certain perturbations whose duration is negligible in comparison with the duration of the process. It has seen considerable development in the last decade; see the monographs of Lakshmikantham et al. [22], Bainov and Simeonov [4], and Samoilenko and Perestyuk [27] where numerous properties of their solutions are studied, and detailed bibliographies are given.

The notion of "nonlocal condition" has been introduced to extend the study of the classical initial value problems; see e.g. [6, 7, 8, 13, 17, 18, 19, 23, 28. It is more precise for describing nature phenomena than the classical condition since more information is taken into account, thereby decreasing the negative effects incurred by a possibly erroneous single measurement taken at the initial time. The study of abstract nonlocal initial value problems was initiated by Byszewski, we refer to some of the papers below. Byszewski [10, 11], Byszewski and Lakshmikantham [9] give the existence and uniqueness of mild solutions and classical solutions when $f$ and $g$ satisfy the Lipschitz -type conditions. Akca et al. [3] initiated the study of impulsive differential equations with nonlocal conditions in Banach spaces. For more details on nonlocal conditions and impulsive differential equations we refer [1, 2, 14, 15, 24].

Recently many authors [17, 23, 29] have been studied the case $A$ is linear, densely defined operator on $X$ which generates a $C_{0}$ - semigroup. Very recently, Z. Fan [19] obtained the existence of mild solutions for the following impulsive semilinear differential equation

$$
\begin{aligned}
u^{\prime}(t) & =A u(t)+f(t, u(t)), \quad 0 \leq t \leq b, \quad t \neq t_{i} \\
u(0) & =u_{0}-g(u), \\
\Delta u\left(t_{i}\right) & =I_{i}\left(u\left(t_{i}\right)\right), \quad i=1,2, \ldots, p, \quad 0<t_{1}<t_{2}<\cdots<t_{p}<b
\end{aligned}
$$

where the author proved the results under the assumptions of Hausdorff's measure of noncompactness. However, as indicated in [16], we some times need to study the nondensely defined operators. It occurs in many situations due to restrictions on the space where the equation is considered or due to boundary conditions. Recently, Z. Fan [18] proved the existence of integral solutions for the following partial differential equations with nonlocal conditions

$$
\begin{aligned}
& u^{\prime}(t)=A u(t)+f(t, u(t)), \quad t \in(0, b] \\
& u(0)=g(u)
\end{aligned}
$$

where the operator $A$ is nondensely defined. The author established the results under the assumptions of the Hausdorff's measure of noncompactness. The present paper is motivated by the recent papers of Z. Fan [18, 19]. We use some hypothesis in [18, 19], and using the method of Hausdorff's measure of noncompactness, we give the existence of integral solution of impulsive differential equation with nonlocal conditions (1.1)(1.3). The results obtained in this paper are generalizations of the results given by [12, 18, 19, 23, 24].

\section{Preliminaries}

In this section, we introduce notations, definitions, and preliminary facts which are used throughout this paper.

Let $(X,\|\cdot\|)$ be a real Banach space. We denote by $C(0, b ; X)$ the space of $X$-valued continuous functions on $[0, b]$ with the norm

$$
\|u\|=\sup \{\|u(t)\|, t \in[0, b]\} .
$$

A measurable function $f:[0, b] \rightarrow X$ is Bochner integrable if and only if $|f|$ is Lebesgue integrable. For properties of the Bochner integrable, see for instance, Yosida [30]. By $L^{1}(0, b ; X)$ the space of $X$-valued 
Bochner integrable functions on $[0, b]$ with the norm

$$
\|f\|_{L^{1}}=\int_{0}^{b}\|f(t)\| d t .
$$

Let $\mathcal{P C}(0, b ; X)=\left\{u:[0, b] \rightarrow X: u(t)\right.$ be continuous at $t \neq t_{i}$ and left continuous at $t=t_{i}$ and the right limit $u\left(t_{i}^{+}\right)$exists for $\left.i=1,2, \ldots, p\right\}$. Evidently $\mathcal{P C}(0, b ; X)$ is a Banach space with the norm

$$
\|u\|_{\mathcal{P C}}=\sup _{t \in[0, b]}\|u(t)\| .
$$

Throughout this work, we suppose that

(H1) The linear operator $A: D(A) \subset X \rightarrow X$ satisfies the Hille-Yosida condition, if there exist $\bar{M} \geq 0$ and $\omega \in R$ such that $(\omega,+\infty) \subset \rho(A)$ and

$$
\sup \left\{(\lambda-\omega)^{n}\left\|\mathbb{R}(\lambda, A)^{n}\right\|: n \in N, \lambda>\omega\right\} \leq \bar{M},
$$

where $\mathbb{R}(\lambda, A)=(\lambda I-A)^{-1}, \rho(A)$ is the resolvent set of $A$.

(H2) The operator $T(t)$ generated by $A_{0}$ is compact in $\overline{D(A)}$ when $t>0$ and $M=\sup _{t \in[0, b]}\|T(t)\|$.

Definition 2.1. A function $u:[0, b] \rightarrow X$ is said to be an integral solution of (1.1)-(1.3) on $[0, b]$ if the following conditions hold:

(i) $u \in \mathcal{P C}(0, b ; X)$;

(ii) $\int_{0}^{s} u(s) d s \in D(A)$ for $t \in[0, b]$;

(iii) $u(t)=g(u)+A \int_{0}^{t} u(s) d s+\int_{0}^{t} f(s, u(s)) d s+\sum_{0<t_{i}<t} I_{i}\left(u\left(t_{i}\right)\right)$, for $t \in[0, b]$.

From the closedness property of $A$, one can see that if $u$ is an integral solution of (1.1)-(1.3) on $[0, b]$, then for all $t \in[0, b], u(t) \in \overline{D(A)}$. In particular, $u(0) \in \overline{D(A)}$.

Let $A_{0}$ be the part of $A$ in $\overline{D(A)}$ defined by

$$
\begin{aligned}
D\left(A_{0}\right) & =\{x \in D(A): A x \in \overline{D(A)}\}, \\
A_{0} x & =A x .
\end{aligned}
$$

Then $A_{0}$ generates a $C_{0}$ - semigroup $\{T(t)\}_{t \geq 0}$ on $\overline{D(A)}$ ( see Pazy [26] for semigroup theory) and the integral solution in Definition 2.1 ( if it exists) is given by

$$
u(t)=T(t) g(u)+\lim _{\lambda \rightarrow+\infty} \int_{0}^{t} T(t-s) B(\lambda) f(s, u(s)) d s+\sum_{0<t_{i}<t} T\left(t-t_{i}\right) I_{i}\left(u\left(t_{i}\right)\right), \quad 0 \leq t \leq b,
$$

where $B(\lambda)=\lambda \mathbb{R}(\lambda ; A)$. For more details about nondensely defined operators and integrated semigroups we refer to [13, 16, 21].

Next, we introduce the Hausdorff's measure of noncompactness $\alpha(\cdot)$ defined on each bounded subset $\Omega$ of Banach space $Y$ by

$$
\alpha(\Omega)=\inf \{\epsilon>0 ; \Omega \text { has a finite } \epsilon-\text { net in } Y\} .
$$

Some basic properties of $\alpha(\cdot)$ are given in the following Lemma. 
Lemma 2.2. ([5]). Let $Y$ be a real Banach space and $B, C \subseteq Y$ be bounded, the following properties are satisfied:

(1) $B$ is pre-compact if and only if $\alpha(B)=0$;

(2) $\alpha(B)=\alpha(\bar{B})=\alpha(\operatorname{conv} B)$, where $\bar{B}$ and conv $B$ mean the closure and convex hull of $B$, respectively;

(3) $\alpha(B) \leq \alpha(C)$ when $B \subseteq C$;

(4) $\alpha(B+C) \leq \alpha(B)+\alpha(C)$, where $B+C=\{x+y ; x \in B, y \in C\}$;

(5) $\alpha(B \cup C) \leq \max \{\alpha(B), \alpha(C)\}$;

(6) $\alpha(\lambda B)=|\lambda| \alpha(B)$ for any $\lambda \in R$;

(7) If the map $Q: D(Q) \subseteq Y \rightarrow Z$ is Lipschitz continuous with constant $k$, then $\alpha(Q B) \leq k \alpha(B)$ for any bounded subset $B \subseteq D(Q)$, where $Z$ be a Banach space.

(8) $\alpha(B)=\inf \left\{d_{Y}(B, C) ; C \subseteq Y\right.$ be pre-compact $\}=\inf \left\{d_{Y}(B, C) ; C \subseteq Y\right.$ be finite valued $\}$, where $d_{Y}(B, C)$ means the nonsymmetric (or symmetric) Hausdorff distance between $B$ and $C$ in $Y$;

(9) If $\left\{W_{n}\right\}_{n=1}^{+\infty}$ is a decreasing sequence of bounded closed nonempty subsets of $Y$ and $\lim _{n \rightarrow \infty} \alpha\left(W_{n}\right)=0$, then $\cap_{n=1}^{+\infty} W_{n}$ is nonempty and compact in $Y$.

The map $Q: W \subseteq Y \rightarrow Y$ is said to be an $\alpha$-contraction if there exists a positive constant $k<1$ such that $\alpha(Q C) \leq k \alpha(C)$ for any bounded closed subset $C \subseteq W$, where $Y$ is a Banach space.

Lemma 2.3. ([5], Darbo-Sadovskii). If $W \subseteq Y$ is bounded closed and convex, the continuous map $Q: W \rightarrow$ $W$ is an $\alpha$-contraction, then the map $Q$ has at least one fixed point in $W$.

Definition 2.4. A countable set $\left\{f_{n}\right\}_{n=1}^{+\infty} \subset L^{1}(0, b ; X)$ is said to be semicompact if the sequence $\left\{f_{n}(t)\right\}_{n=1}^{+\infty}$ is compact in $X$ for a.e. $t \in[0, b]$ and if there is a function $\mu \in L^{1}(0, b ; \mathbb{R})$ satisfying $\sup _{n \geq 1}\left\|f_{n}(t)\right\| \leq \mu(t)$ for a.e. $t \in[0, b]$.

Definition 2.5. We call the operator $G: L^{1}(0, b ; X) \rightarrow \mathcal{P C}(0, b ; X)$ defined by

$$
G f(t)=\lim _{\lambda \rightarrow+\infty} \int_{0}^{t} T(t-s) B(\lambda) f(s) d s, \quad t \in[0, b]
$$

as the Cauchy operator.

Now, we give the following properties about the Cauchy operator $G$.

Proposition 2.6. ([20]) Let $G$ be the Cauchy operator defiend by (2.1), $\left\{f_{n}\right\}_{n=1}^{+\infty}$ a sequence of functions in $L^{1}(0, b ; X)$. Assume that there exist $\mu, \eta$ in $L^{1}(0, b ; \mathbb{R})$ satisfying

$$
\sup _{n \geq 1}\left\|f_{n}(t)\right\| \leq \mu(t) \quad \text { and } \quad \alpha\left(\left\{f_{n}(t)\right\}_{n=1}^{+\infty}\right) \leq \eta(t) \text { a.e. } t \in[0, b] .
$$

Then for all $t \in[0, b]$, we have

$$
\alpha\left(\left\{\left(G f_{n}\right)(t)\right\}_{n=1}^{+\infty}\right) \leq 2 M \bar{M} \int_{0}^{t} \eta(s) d s
$$

where $\alpha$ is the Hausdorff measure of noncompactness.

Proposition 2.7. ([20]) Let $G$ be the Cauchy operator defined by (2.1). Then for every semicompact set $\left\{f_{n}\right\}_{n=1}^{+\infty} \subset L^{1}(0, b ; X)$, the set $\left\{G f_{n}\right\}_{n=1}^{+\infty}$ is relatively compact in $\mathcal{P C}(0, b ; X)$. 
Proposition 2.8. If $D \subseteq \mathcal{P C}(0, b ; X)$ be bounded, then we have

$$
\sup _{t \in[0, b]} \alpha(D(t)) \leq \alpha(D)
$$

The follwoing fixed point theorem, a nonlinear alternative of Monch type, plays a crucial role in our existence of integral solutions of (1.1)-(1.3).

Theorem 2.9. ([25]) Let $E$ be a Banach space, $U$ an open subset of $E$ and $0 \in U$. Suppose that $F: \bar{U} \rightarrow E$ is a continuous map which satisfies Monch's condition ( that is, if $D \subseteq \bar{U}$ is countable and $D \subseteq \overline{c o}(\{0\} \cup F(D)$ ), then $\bar{D}$ is compact) and assume that

$$
x \neq \lambda F(x) \quad \text { for } x \in \partial U \text { and } \lambda \in(0,1)
$$

holds. Then $F$ has a fixed point in $\bar{U}$.

\section{Existence Results}

In this section, we present and prove the existence of integral solutions for the impulsive nonlocal problem (1.1)-(1.3).

Let $r$ be a finite positive constant. We consider the sets $B_{r}=\{x \in X:\|x\| \leq r\}, W_{r}=\{u \in$ $\left.\mathcal{P C}(0, b ; X): u(t) \in B_{r}, \forall t \in[0, b]\right\}$. 
Now, we list the following hypotheses:

(H3) The operator $T(t), 0 \leq t \leq b$ generated by $A_{0}$ is equicontinuous;

(H4) (i) $f:[0, b] \times X \rightarrow X$, for a.e. $t \in[0, b]$, the function $f(t, \cdot): X \rightarrow X$ is continuous for all $x \in X$, the function $f(\cdot, x):[0, b] \rightarrow X$ is measurable.

(ii) Moreover, for any $l>0$, there exists a function $\rho_{l} \in L^{1}(0, b ; \mathbb{R})$ such that

$$
\|f(t, x)\| \leq \rho_{l}(t)
$$

for a.e. $t \in[0, b]$ and all $x \in B_{l}$;

(iii) There exists a function $m \in L^{1}(0, b ; \mathbb{R})$ and a nondecreasing continuous function $\psi:[0, \infty) \rightarrow$ $(0, \infty)$ such that

$$
\|f(t, x)\| \leq m(t) \psi(\|x\|)
$$

for all $x \in X, t \in[0, b]$.

(H5) There exists a function $h \in L^{1}(0, b ; \mathbb{R})$ such that for every bounded $D \subseteq W_{r}$,

$$
\alpha(f(t, D)) \leq h(t) \alpha(D)
$$

for a.e. $t \in[0, b]$, where $\alpha$ is the Hausdorff measure of noncompactness.

(H6) $g: \mathcal{P C}(0, b ; X) \rightarrow \overline{D(A)}$ is Lipschitz continuous with Lipschitz constant k;

(H7) $I_{i}: X \rightarrow \overline{D(A)}$ is Lipschitz continuous with Lipschitz constant $k_{i}, i=1,2, \ldots, p$.

Theorem 3.1. Assume that the conditions (H1),(H2), (H4)(i)(ii) and (H6)-(H7) are satisfied. Then the nonlocal impulsive problem (1.1)-(1.3) has at least one integral solution on $[0, b]$ provided that

$$
M\left[\left(k+\sum_{i=1}^{p} k_{i}\right) r+\|g(0)\|+\sum_{i=1}^{p}\left\|I_{i}(0)\right\|+\bar{M}\left\|\rho_{r}\right\|_{L^{1}}\right] \leq r
$$

Proof. Define the operator $Q: \mathcal{P C}(0, b ; X) \rightarrow \mathcal{P C}(0, b ; X)$ by

$$
(Q u)(t)=\left(Q_{1} u\right)(t)+\left(Q_{2} u\right)(t)+\left(Q_{3} u\right)(t),
$$

with

$$
\begin{aligned}
& \left(Q_{1} u\right)(t)=T(t) g(u), \\
& \left(Q_{2} u\right)(t)=\sum_{0<t_{i}<t} T\left(t-t_{i}\right) I_{i}\left(u\left(t_{i}\right)\right), \\
& \left(Q_{3} u\right)(t)=\lim _{\lambda \rightarrow+\infty} \int_{0}^{t} T(t-s) B(\lambda) f(s, u(s)) d s,
\end{aligned}
$$

for all $t \in[0, b]$. It is easy to see that the fixed point of $Q$ is the integral solution of the nonlocal impulsive problem (1.1)-(1.3). Subsequently, we will prove that $Q$ has a fixed point, by using Lemma 2.2.

Firstly, we prove that the mapping $Q$ is continuous on $\mathcal{P C}(0, b ; X)$. For this purpose, let $\left\{u_{n}\right\}_{n=1}^{+\infty}$ be a sequence in $\mathcal{P C}(0, b ; X)$ with $\lim _{n \rightarrow \infty} u_{n}=u$ in $\mathcal{P C}(0, b ; X)$. By the continuity of $f$ with respect to the second argument, we deduce that for each $s \in[0, b], f\left(s, u_{n}(s)\right)$ converges to $f(s, u(s))$ in $X$; and we have

$$
\begin{aligned}
\left\|Q u_{n}-Q u\right\|_{\mathcal{P C}} \leq & M\left[\left\|g\left(u_{n}\right)-g(u)\right\|+\sum_{i=1}^{p}\left\|I_{i}\left(u_{n}\left(t_{i}\right)\right)-I_{i}\left(u\left(t_{i}\right)\right)\right\|\right] \\
& +M \bar{M} \int_{0}^{b}\left\|f\left(s, u_{n}(s)\right)-f(s, u(s))\right\| d s .
\end{aligned}
$$


Then by continuity of $g, I_{i}$ and using dominated convergence theorem, we get $\lim _{n \rightarrow \infty} Q u_{n}=Q u$ in $\mathcal{P C}(0, b ; X)$, which implies that the mapping $Q$ is continuous on $\mathcal{P C}(0, b ; X)$.

Secondly, we claim that $Q W_{r} \subseteq W_{r}$. In fact, for any $u \in W_{r} \subseteq \mathcal{P C}(0, b ; X)$, by (H4)(ii), (3.1) and

$$
\|B(\lambda)\| \leq \frac{\lambda \bar{M}}{\lambda-\omega} \rightarrow \bar{M}, \quad \text { as } \lambda \rightarrow+\infty .
$$

We have

$$
\begin{aligned}
\|(Q u)(t)\| \leq & \|T(t) g(u)\|+\left\|\lim _{\lambda \rightarrow+\infty} \int_{0}^{t} T(t-s) B(\lambda) f(s, u(s)) d s\right\|+\left\|\sum_{0<t_{i}<t} T\left(t-t_{i}\right) I_{i}\left(u\left(t_{i}\right)\right)\right\| \\
\leq & M[\|g(u)-g(0)\|+\|g(0)\|]+M \bar{M} \int_{0}^{b}\|f(s, u(s))\| d s \\
& +M\left[\sum_{i=1}^{p}\left\|I_{i}\left(u\left(t_{i}\right)\right)-I_{i}(0)\right\|+\left\|I_{i}(0)\right\|\right] \\
\leq & M\left[\left(k+\sum_{i=1}^{p} k_{i}\right) r+\|g(0)\|+\sum_{i=1}^{p}\left\|I_{i}(0)\right\|+\bar{M}\left\|\rho_{r}\right\|_{L^{1}}\right] \\
\leq & r
\end{aligned}
$$

It implies that $Q W_{r} \subseteq W_{r}$.

Now, according to Lemma 2.2, it remains to prove that $Q$ is an $\alpha$-contraction in $W_{r}$. By using conditions (H6) and (H7), we get that $Q_{1}+Q_{2}: W_{r} \rightarrow \mathcal{P C}(0, b ; X)$ is Lipschitz continuous with constant $M\left(k+\sum_{i=1}^{p} k_{i}\right)$. In fact, for any $u, v \in W_{r}$, we have

$$
\begin{aligned}
\left\|\left(Q_{1}+Q_{2}\right) u-\left(Q_{1}+Q_{2}\right) v\right\|_{\mathcal{P C}} \leq & \sup _{t \in[0, b]}[\|T(t)[g(u)-g(v)]\| \\
& \left.+\sum_{i=1}^{p}\left\|T\left(t-t_{i}\right)\left[I_{i}\left(u\left(t_{i}\right)\right)-I_{i}\left(v\left(t_{i}\right)\right)\right]\right\|\right] \\
\leq & M\left[k\|u-v\|_{\mathcal{P C}}+\sum_{i=1}^{p} k_{i}\|u-v\|_{\mathcal{P C}}\right] \\
= & M\left[k+\sum_{i=1}^{p} k_{i}\right]\|u-v\|_{\mathcal{P C}}
\end{aligned}
$$

Thus, Lemma $2.1(7)$, we obtain that

$$
\alpha\left(\left(Q_{1}+Q_{2}\right) W_{r}\right) \leq M\left[k+\sum_{i=1}^{p} k_{i}\right] \alpha\left(W_{r}\right) .
$$

Finally, we prove that $Q_{3}: W_{r} \rightarrow \mathcal{P C}(0, b ; X)$ is a compact operator by using Arzela- Ascoli's theorem. From [18, Theorem 3.1], we see that $Q_{3}$ is compact. Thus $\alpha\left(Q_{3} W_{r}\right)=0$. Consequently,

$$
\begin{aligned}
\alpha\left(Q W_{r}\right) & \leq \alpha\left(\left(Q_{1}+Q_{2}\right) W_{r}\right)+\alpha\left(Q_{3} W_{r}\right) \\
& \leq M\left[k+\sum_{i=1}^{p} k_{i}\right] \alpha\left(W_{r}\right)
\end{aligned}
$$

Since the condition (3.1), $M\left[k+\sum_{i=1}^{p} k_{i}\right]<1$, the mapping $Q$ is an $\alpha$-contraction in $W_{r}$. By DarboSadovskii's fixed point theorem, the operator $Q$ has a fixed point in $W_{r}$, which is the integral solution of the nonlocal impulsive problem (1.1)-(1.3). This completes the proof. 
Theorem 3.2. Assume that the conditions (H1),(H2), (H4)(i)(iii) and (H5)-(H7) are satisfied. Then the nonlocal impulsive problem (1.1)-(1.3) has at least one integral solution on $[0, b]$ provided that there exists a constant $N>0$ with

$$
\frac{\left[1-M\left(k+\sum_{i=1}^{p} k_{i}\right)\right] N}{M\left[\|g(0)\|+\bar{M} \psi(N)\|m\|_{L^{1}}+\sum_{i=1}^{p}\left\|I_{i}(0)\right\|\right]}>1
$$

and that

$$
M\left[\left(k+\sum_{i=1}^{p} k_{i}\right)+2 \bar{M}\|h\|_{L^{1}}\right]<1 .
$$

Proof. We define the operator $Q$ as defined in (3.2)-(3.5) for all $t \in[0, b]$. It is easy to see that the fixed point of $Q$ is the integral solution of nonlocal impulsive problem (1.1)-(1.3). Subsequently, we will prove that $Q$ has a fixed point by using Theorem 2.1. For better readability, we break the proofs into three steps. Step 1: The operator $Q$ is continuous on $\mathcal{P C}(0, b ; X)$.

For this purpose, let $\left\{u_{n}\right\}_{n=1}^{+\infty}$ be a sequence in $\mathcal{P C}(0, b ; X)$ with $\lim _{n \rightarrow \infty} u_{n}=u$ in $\mathcal{P C}(0, b ; X)$. Then by (H4)(i), we have that

$$
f\left(s, u_{n}(s)\right) \rightarrow f(s, u(s)), \quad(n \rightarrow+\infty) \quad \forall s \in[0, b] .
$$

Since $I_{i}, i=1,2, \ldots, p$ is continuous and $\left\|f\left(s, u_{n}(s)\right)-f(s, u(s))\right\| \leq 2 \psi(N) m(s)$ for some integer $N$, by (H4)(iii) and (H6)-(H7) together with the dominated convergence theorem, we have

$$
\begin{aligned}
\left\|Q u_{n}-Q u\right\|_{\mathcal{P C}} \leq & M\left[\left\|g\left(u_{n}\right)-g(u)\right\|+\sum_{i=1}^{p}\left\|I_{i}\left(u_{n}\left(t_{i}\right)\right)-I_{i}\left(u\left(t_{i}\right)\right)\right\|\right] \\
& +M \bar{M} \int_{0}^{b}\left\|f\left(s, u_{n}(s)\right)-f(s, u(s))\right\| d s \\
& \rightarrow 0 \text { as } n \rightarrow \infty .
\end{aligned}
$$

Thus, $Q$ is continuous on $\mathcal{P C}(0, b ; X)$.

Step 2: The Monch's condition holds.

For this purpose, let $D \subseteq W_{r}$ be countable and $D \subseteq \overline{c o}(\{0\} \cup Q(D))$. We show that $\alpha(D)=0$. Without loss of generality, we may assume that $D=\left\{u_{n}\right\}_{n=1}^{+\infty}$. By using the condition (H3), we can easily verify that $\left\{Q_{3} u_{n}\right\}_{n=1}^{+\infty}$ is equicontinuous. Moreover, $Q_{1}+Q_{2}: D \rightarrow \mathcal{P C}(0, b ; X)$ is Lipschitz continuous with constant $M\left(k+\sum_{i=1}^{p} k_{i}\right)$ due to the conditions (H6) and (H7). In fact, $u, v \in D$, we have

$$
\begin{aligned}
\left\|\left(Q_{1}+Q_{2}\right) u-\left(Q_{1}+Q_{2}\right) v\right\|_{\mathcal{P C}} \leq & \sup _{t \in[0, b]}[\|T(t)[g(u)-g(v)]\| \\
& \left.+\sum_{i=1}^{p}\left\|T\left(t-t_{i}\right)\left[I_{i}\left(u\left(t_{i}\right)\right)-I_{i}\left(v\left(t_{i}\right)\right)\right]\right\|\right] \\
\leq & M\left[k\|u-v\|_{\mathcal{P C}}+\sum_{i=1}^{p} k_{i}\|u-v\|_{\mathcal{P C}}\right] \\
= & M\left[k+\sum_{i=1}^{p} k_{i}\right]\|u-v\|_{\mathcal{P C}}
\end{aligned}
$$


So, from Proposition (2.1),(2.3) and Lemma 2.1, we have

$$
\begin{aligned}
\alpha\left(\left\{Q u_{n}\right\}_{n=1}^{+\infty}\right) \leq & \alpha\left(\left\{\left(Q_{1}+Q_{2}\right) u_{n}\right\}_{n=1}^{+\infty}\right)+\alpha\left(\left\{Q_{3} u_{n}\right\}_{n=1}^{+\infty}\right) \\
\leq & M\left(k+\sum_{i=1}^{p} k_{i}\right) \alpha\left(\left\{u_{n}\right\}_{n=1}^{+\infty}\right) \\
& +\sup _{t \in[0, b]} \alpha\left(\left\{\lim _{\lambda \rightarrow+\infty} \int_{0}^{t} T(t-s) B(\lambda) f\left(s, u_{n}(s)\right) d s\right\}_{n=1}^{+\infty}\right) \\
\leq & M\left(k+\sum_{i=1}^{p} k_{i}\right) \alpha\left(\left\{u_{n}\right\}_{n=1}^{+\infty}\right) \\
& +2 M \bar{M} \int_{0}^{b} h(s) \sup _{t \in[0, b]} \alpha\left(\left\{u_{n}(t)\right\}_{n=1}^{+\infty}\right) d s \\
\leq & M\left(k+\sum_{i=1}^{p} k_{i}\right) \alpha\left(\left\{u_{n}\right\}_{n=1}^{+\infty}\right)+2 M \bar{M}\|h\|_{L^{1}} \alpha\left(\left\{u_{n}\right\}_{n=1}^{+\infty}\right) \\
\leq & M\left[\left(k+\sum_{i=1}^{p} k_{i}\right)+2 \bar{M}\|h\|_{L^{1}}\right] \alpha\left(\left\{u_{n}\right\}_{n=1}^{+\infty}\right)
\end{aligned}
$$

Thus, we get that

$$
\alpha(D) \leq \alpha(\overline{c o}\{0\} \cup Q(D))=\alpha(Q(D)) \leq M\left[\left(k+\sum_{i=1}^{p} k_{i}\right)+2 \bar{M}\|h\|_{L^{1}}\right] \alpha(D)
$$

which implies that $\alpha(D)=0$, since the condition (3.7) holds.

Step 3: Now, let $\mu \in(0,1)$ and $u=\mu Q(u)$. Then for $t \in[0, b]$, we have

$$
u(t)=\mu T(t) g(u)+\mu \lim _{\lambda \rightarrow+\infty} \int_{0}^{t} T(t-s) B(\lambda) f(s, u(s)) d s+\mu \sum_{0<t_{i}<t} T\left(t-t_{i}\right) I_{i}\left(u\left(t_{i}\right)\right)
$$

and one has

$$
\begin{aligned}
\|u(t)\| \leq & M[\|g(u)-g(0)\|+\|g(0)\|]+M \bar{M} \int_{0}^{b}\|f(s, u(s))\| d s \\
& +M\left[\sum_{i=1}^{p}\left\|I_{i}\left(u\left(t_{i}\right)\right)-I_{i}(0)\right\|+\left\|I_{i}(0)\right\|\right] \\
\leq & M[k\|u\|+\|g(0)\|]+M \bar{M} \int_{0}^{b} m(s) \psi(\|u\|) d s+M \sum_{i=1}^{p}\left[k_{i}\|u\|+\left\|I_{i}(0)\right\|\right]
\end{aligned}
$$

Consequently,

$$
\frac{\left[1-M\left(k+\sum_{i=1}^{p} k_{i}\right)\right]\|u\|}{M\left[\|g(0)\|+\bar{M} \psi(\|u\|)\|m\|_{L^{1}}+\sum_{i=1}^{p}\left\|I_{i}(0)\right\|\right]} \leq 1
$$

Then by (3.6) there exists $N$ such that $\|u\| \neq N$. Set

$$
U=\{u \in \mathcal{P C}(0, b ; X):\|u\| \leq N\} .
$$

From the choice of $U$ there is no $u \in \partial U$ such that $u=\mu Q(u)$ for some $\mu \in(0,1)$. Thus, we get a fixed point $Q$ in $\bar{U}$ due to Theorem 2.1, which is a integral solution of (1.1)-(1.3). 


\section{Example}

As an application of Theorem 3.1, we consider the following system

$$
\begin{aligned}
\frac{\partial}{\partial t} w(t, x) & =\nabla w(t, x)+F(t, w(t, x)), 0 \leq t \leq b, t \neq t_{i}, i=1,2, \ldots, p, x \in \Omega, \\
w\left(t_{i}^{+}, x\right) & -w\left(t_{i}^{-}, x\right)=I_{i}\left(w\left(t_{i}, x\right)\right), \quad i=1,2, \ldots, p \\
w(t, x) & =0, \quad 0 \leq t \leq b, \quad x \in \partial \Omega \\
w(0, x) & +\sum_{j=1}^{p} c_{j} w\left(t_{j}, x\right)=u_{0}(x), \quad 0 \leq t_{j} \leq b, \quad x \in \Omega
\end{aligned}
$$

where $\Omega$ is a bounded open set in $R^{n}$ with regular boundary $\partial \Omega, u_{0} \in C\left(\bar{\Omega} ; R^{n}\right), \quad \nabla=\sum_{k=1}^{n} \frac{\partial^{2}}{\partial x_{k}^{2}}, F$ : $[0, b] \times \bar{\Omega} \rightarrow R^{n}, c_{j}, j=1,2, \ldots, p$ and $I_{i}, i=1,2, \ldots, p$ are given real numbers.

We choose $X=C\left(\bar{\Omega}, R^{n}\right)$ and we consider the operator $A: D(A) \subseteq X \rightarrow X$ defined by

$$
\begin{aligned}
D(A) & =\{z \in X: \nabla z \in X \quad \text { and } z=0 \text { on } \partial \Omega\}, \\
A z & =\nabla z .
\end{aligned}
$$

Now, we have

(i) $\overline{D(A)}=C_{0}\left(\bar{\Omega} ; R^{n}\right)=\{z \in X: z=0$ on $\partial \Omega\} \neq X$;

(ii) $(0,+\infty) \subseteq \rho(A)$;

(iii) $\|\mathbb{R}(\lambda ; A)\| \leq \frac{1}{\lambda}, \quad$ for $\lambda>0$.

This implies that the operator $A$ satisfies the conditions (H1) and (H2) with $M=\bar{M}=1$.

We assume that

(1) $f:[0, b] \times X \rightarrow X$ is a continuous function defined by

$$
f(t, z)(x)=F(t, z(x)), \quad x \in \bar{\Omega} .
$$

(2) $g: \mathcal{P C}(0, b ; X) \rightarrow X$ is a continuous function defined by

$$
g(u)(x)=u_{0}(x)-\sum_{j=1}^{p} c_{j} u\left(t_{j}\right)(x), \quad t \in[0, b], \quad x \in \bar{\Omega},
$$

where $u(t)(x)=w(t, x), \quad t \geq 0, x \in \bar{\Omega}$.

(3) $I_{i}: X \rightarrow X$ is a continuous function defined by

$$
\Delta w\left(t_{i}, x\right)=w\left(t_{i}^{+}, x\right)-w\left(t_{i}^{-}, x\right), \quad i=1,2, \ldots, p,
$$

where $u\left(t_{i}\right)(x)=w\left(t_{i}, x\right), \quad t \geq 0, x \in \bar{\Omega}$.

Under these assumptions, the problem (4.1)-(4.4) can be reformulated as the abstract problem (1.1)-(1.3).

Thus, under the appropriate conditions on the functions $f, g$ and $I_{i}$ as those in (H4)(i)(ii) and (H6)-(H7), the problem (1.1)-(1.3) has an integral solution.

\section{Acknowledgements}

The authors dedicate this paper to Silver Jubilee Year Celebrations of Karunya University, Coimbatore641 114, Tamil Nadu, India. And also the authors wish to thank Dr. Paul Dhinakaran, Chancellor, Dr. Paul P. Appasamy, ViceChancellor, and Dr(Mrs). Anne Mary Fernandez, Registrar, of Karunya University, Coimbatore, for their constant encouragements and support for this research work. 


\section{References}

[1] A. Anguraj and M. Mallika Arjunan, Existence and uniqueness of mild and classical solutions of impulsive evolution equations, Electronic Journal of Differential Equations, 111 (2005), 1-8. 1

[2] A. Anguraj and M. Mallika Arjunan, Existence results for an impulsive neutral integro-differential equations in Banach spaces, Nonlinear Studies, 16(1) (2009), 33-48. 1

[3] H. Akca, A. Boucherif and V. Covachev, Impulsive functional differential equations with nonlocal conditions, Inter. J. Math. Math. Sci., 29:5 (2002), 251-256. 1

[4] D.D. Bainov and P.S. Simeonov, Impulsive Differential Equations: Periodic Solutions and Applications, Longman Scientific and Technical Group, England, 1993. 1]

[5] J. Banas and K. Goebel, Measure of Noncompactness in Banach Spaces, in: Lecture Notes in Pure and Appl. Math., 60, Marcel Dekker, New York, 1980. 2.2 2.3

[6] M. Benchohra and S.K. Ntouyas, Existence of mild solutions for certain delay semilinear evolution inclusions with nonlocal condition, Dynam. Systems Appl., 9:3 (2000), 405-412. 1

[7] M. Benchohra and S.K. Ntouyas, Existence of mild solutions of semilinear evolution inclusions with nonlocal conditions, Georgian Math. J., 7 (2000), 221-230. 1

[8] M. Benchohra and S.K. Ntouyas, Existence and controllability results for nonlinear differential inclusions with nonlocal conditions, J. Appl. Anal., 8 (2002), 31-46. 1

[9] L. Byszewski and V. Lakshmikantham, Theorems about the existence and uniqueness of solutions of a nonlocal Cauchy problem in Banach spaces, Appl. Anal., 40 (1990), 11-19. 1

[10] L. Byszewski, Uniqueness criterian for solution to abstract nonlocal Cauchy problem, J. Appl. Math. Stochastic Anal., 162 (1991), 49-54. 1

[11] L. Byszewski, Existence and uniqueness of solutions of a semilinear evolution nonlocal Cauchy problem, Zesz. Nauk. Pol. Rzes. Mat. Fiz., 18 (1993), 109-112. 1

[12] T. Cardinali and P. Rubbioni, Impulsive semilinear differential inclusion: Topological structure of the solution set and solutions on non-compact domains, Nonlinear Anal., 14 (2008), 73-84. 1

[13] Y.K. Chang, A. Anguraj and M. Mallika Arjunan, Existence results for non-densely defined neutral impulsive differential inclusions with nonlocal conditions, J. Appl. Math. Comput., 28 (2008), 79-91. 1,2

[14] Y.K. Chang, A. Anguraj and M. Mallika Arjunan, Existence results for impulsive neutral functional differential equations with infinite delay, Nonlinear Anal.: Hybrid Systems, 2(1) (2008), 209-218. 1

[15] Y.K. Chang, V. Kavitha and M. Mallika Arjunan, Existence results for impulsive neutral differential and integrodifferential equations with nonlocal conditions via fractional operators, Nonlinear Anal.: Hybrid Systems, 4(1) (2010), 32-43. 1

[16] G. Da Prato and E. Sinestrari, Differential operators with non-dense domain, Ann. Scuola Norm. Sup. Pisa Sci., 14 (1987), 285-344. 12

[17] Z. Fan, Q. Dong and G. Li, Semilinear differential equations with nonlocal conditions in Banach spaces, International Journal of Nonlinear Science, Vol. 2(3) (2006), 131-139. 1

[18] Z. Fan, Existence of nondensely defined evolution equations with nonlocal conditions, Nonlinear Anal., 70 (2009), 3829-3836. 1,3

[19] Z. Fan, Impulsive problems for semilinear differential equations with nonlocal conditions, Nonlinear Anal., 72(2) (2010), 1104-1109. 1

[20] M. Kamenskii, V. Obukhovskii and P. Zecca, Condensing Multivalued Maps and Semilinear Differential Inclusions in Banach Spaces: De Gruyter Ser: Nonlinear Anal. Appl., 7, de Gruyter, Berlin, 2001. 2.6, 2.7

[21] H. Kellerman and M. Heiber, Integrated semigroups, J. Funct. Anal., 84 (1989), 160-180. 2

[22] V. Lakshmikantham, D.D. Bainov and P.S. Simeonov, Theory of Impulsive Differential Equations, World Scientific, Singapore, 1989. 1

[23] J. Liang, J.H. Liu and Ti-Jun Xiao, Nonlocal impulsive problems for nonlinear differential equations in Banach spaces, Math. Comput. Model., 49 (2009), 798-804. 1

[24] J.H. Liu, Nonlinear impulsive evolution equations, Dynam. Contin. Discrete Impuls. Sys., 6 (1999), 77-85. 1

[25] H. Monch, Boundary value problems for nonlinear ordinary differential equations of second order in Banach spaces, Nonlinear Anal., 4 (1980), 985-999. 2.9

[26] A. Pazy, Semigroups of linear operators and applications to partial differential equations, Springer-Verlag, Newyork, 1983. 2

[27] A.M. Samoilenko and N.A Perestyuk, Impulsive Differential Equations, World Scientific, Singapore, 1995. 1

[28] B. Selvaraj, M. Mallika Arjunan and V. Kavitha, Existence of solutions for impulsive nonlinear differential equations with nonlocal conditions, J. Korean Society for Industrial and Applied Mathematics, 13(3) (2009), 203-215. 1

[29] X. Xue, Existence of solutions for semilinear nonlocal Cauchy problems in Banach spaces, Electronic Journal of Differential Equations, 64 (2005), 1-7. 1

[30] K. Yosida, Functional Analysis, 6th edn., Springer, Berlin, 1980. 2 